\title{
Cavity-enhanced toroidal dipole force traps for dark-field seeking species
}

\author{
Tim Freegarde ${ }^{\mathrm{a}, *}$, Kishan Dholakia ${ }^{\mathrm{b}}$ \\ ${ }^{a}$ Dipartimento di Fisica, Università di Trento, Via Sommarive 14, Povo TN 38050, Italy \\ ${ }^{\mathrm{b}}$ JF Allen Physics Research Laboratories, School of Physics and Astronomy, University of St. Andrews, North Haugh, \\ St. Andrews, Fife KY16 9SS, Scotland, UK
}

Received 24 July 2001; received in revised form 15 October 2001; accepted 1 November 2001

\begin{abstract}
Dipole force traps for dark-field seeking states of atoms and molecules require regions of low intensity that are completely surrounded by a brighter optical field. Confocal cavities allow the resonant enhancement of these interesting transverse mode superpositions, and put deep far-off-resonance traps within reach of low-power diode lasers. In this paper, we show how an array of dark-field rings may be created simply using a single Gaussian beam. Such a geometry lends itself to the study of toroidal Bose-Einstein condensates. (c) 2002 Elsevier Science B.V. All rights reserved.
\end{abstract}

\section{Introduction}

The optical scattering force is routinely used to cool, confine and manipulate atoms [1], but it is not suitable for species whose spectral complexity causes population loss from the trapping transitions. The dipole force [2] in principle avoids such effects. It can act towards or away from regions of high intensity, depending upon the sign of the detuning of the laser from the radiative transition, and incoherent scattering is reduced by moving far from resonance $[3,4]$, where spontaneous emission is low. In most applications, the laser has been detuned to the red, so that the atoms are attracted towards the intense focus of the laser beam [5].

\footnotetext{
${ }^{*}$ Corresponding author. Fax: +39-0461-881-696.

E-mail address: tim.freegarde@physics.org (T. Freegarde).
}

Despite some residual scattering, such schemes have been used to produce an atomic Bose-Einstein condensate (BEC) [6], to allow the controlled delivery of single atoms [7], and to trap caesium dimers [8].

When the laser is instead detuned to the blue of the radiative transition, the species seek dark, lowfield regions where they are substantially undisturbed; the reduced rate of spontaneous scattering allows storage times of the order of a second, which are sufficient for most experiments [9]. For the dark-field seeking force to provide confinement, however, we require regions of low intensity that are completely surrounded by a brighter optical field. Such a field is not offered by a simple $\mathrm{TEM}_{00}$ laser beam and various schemes have instead been adopted, using sheets of light [10] or the time-average of a rotating laser beam [11]. Zemánek and Foot [12] have shown how a line of 
traps might be formed by the interference between counter-propagating Gaussian beams of differently sized but coincident waists. Other schemes use complex beams such as Laguerre-Gaussians and their superpositions, produced using cylindrical optics [13,14], waveplates [9] or, more robustly, holographic mode-converters $[15,16]$.

Far-off-resonance dipole force traps need high laser intensities to offset the weakness of the interaction and, as the optical absorption is small, resonant cavities have been used to enhance the trapping beams [17-19]. Single standing-wave cavity modes are however suitable only for reddetuned (high-field seeking) traps, for the exact cancellation of the forward and backward travelling beams produces nodal surfaces of zero intensity along which dark-field seeking species would escape. Laguerre-Gaussian modes of a travellingwave cavity allow confinement in two dimensions [20-23], although the translational symmetry prohibits intrinsic axial confinement, which must be provided by additional beams [14].

We show here that a three-dimensional bluedetuned trapping field could be both produced and enhanced by a confocal standing-wave cavity, whose alignment proves not to be unduly critical. As shown in Fig. 1, a single Gaussian laser beam is focussed into the cavity to form at its centre a waist somewhat larger than that of the fundamental cavity mode; the waist of the backward travelling beam, formed by subsequent reflection at the second cavity mirror, is then smaller than the mode waist by the same factor. These beams correspond to superpositions of the cavity modes which, when the cavity is confocal, are simultaneously resonant, and it is destructive interference between them that generates the intensity minima of the trap. Our scheme is inspired by the arrangement suggested by Zemánek and Foot [12] but, where they suggested equal axial intensities, the two beams here are of equal power. This modification dramatically changes the trapping volumes from axial wells into a series of coaxial rings, spaced half a wavelength apart. This is, to the best of our knowledge, the first method to be proposed for the realization of a dipole force trap with a blue-detuned toroidal geometry, and is achieved using simple concave mirrors and a single Gaussian beam.

The cavity-enhanced dipole force has already been applied to studies of cavity quantum electrodynamics $[17,18,24]$ and quantum computing [7,25]. Dissipative interaction with the cavity field has also been proposed as a mechanism for cooling the confined sample [26-28]. The use of confocal cavities would allow the dark-field seeking dipole force to be applied to such studies. The specific trapping geometry described here could be useful for the investigation of persistent currents and vortices in quantum degenerate gases [29], as well as for studies of the coupling between adjacent microtraps.

The spectral complexity of molecular species presents particular challenges for optical trapping, for residual scattering may remove population catastrophically and, thanks to the distribution
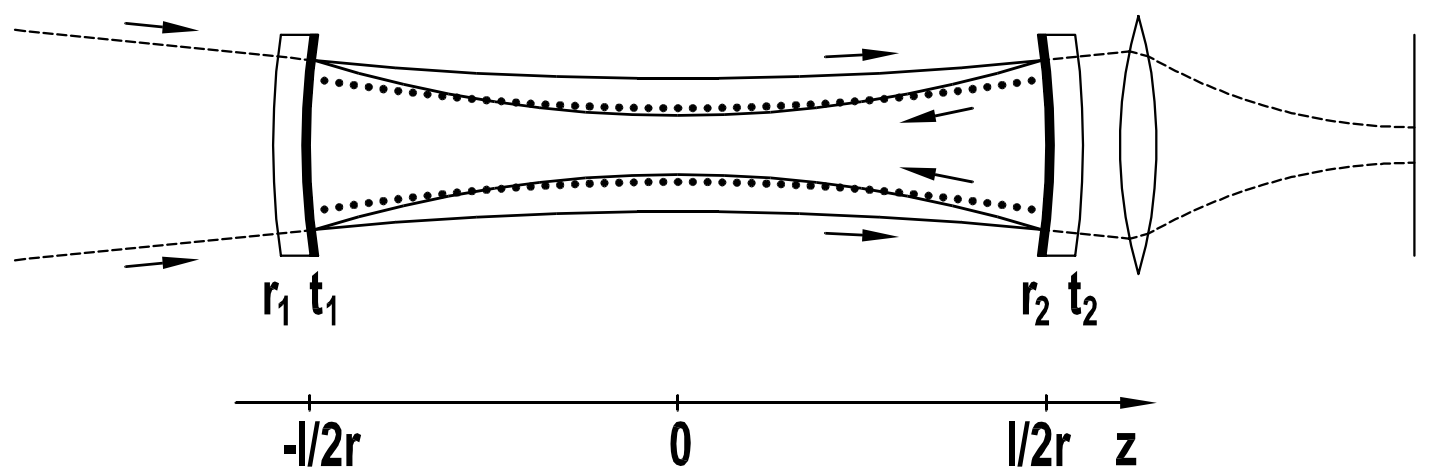

Fig. 1. A confocal resonator supports modes which superpose to form Gaussian beams whose waist sizes depend upon the direction of propagation. The positions of the trap minima are indicated by the dotted lines. 
across rotational states, the trapping laser cannot be brought as close to resonance as with atomic candidates. Molecules have therefore hitherto been trapped only using the red-detuned force of powerful mainframe lasers [8]: with cavity enhancement, they could be confined in less perturbing blue-detuned potentials using only modest laser powers. Indeed, cavity enhancement allows the trapping field to be tuned further from resonance for any species, thus offering lower scattering rates for the same trap depth. In the scheme presented here, the trap minima lie on a surface which falls throughout within the fundamental Gaussian mode of the same cavity, so the same mirrors could be used to detect the trapped species using cavity-enhanced techniques [30,31].

\section{Analysis}

The electric field of a normalized LaguerreGaussian beam of order $(p, m)$, waist radius $w(0)$ and wavelength $\lambda$, propagating in a positive direction along the $z$-axis, may be written in terms of the wavenumber $k=2 \pi / \lambda$ and Rayleigh range $z_{R}=\pi w_{0}^{2} / \lambda$ by

$$
\begin{aligned}
& \mathscr{E}_{p m}(r, z) \\
& =\sqrt{\frac{4 p !}{\left(1+\delta_{0 m}\right) \pi(p+|m|) !}} L_{p}^{|m|}\left(\frac{2 r^{2}}{w(z)^{2}}\right) \\
& \times \frac{\exp \left(\mathrm{i}(2 p+|m|+1) \tan ^{-1}\left(z / z_{R}\right)\right)}{w(z)}\left(\frac{\sqrt{2} r}{w(z)}\right)^{|m|} \\
& \quad \times \exp \left(-\frac{r^{2}}{w(z)^{2}}-\frac{\mathrm{i} k r^{2}}{2 R(z)}+\mathrm{i} m \theta\right)
\end{aligned}
$$

where $w(z)=w(0) \sqrt{1+\left(z / z_{R}\right)^{2}}, \quad R(z)=z+z_{R}^{2} / z$. Such beams, which have $p$ off-axis nodes as a function of radius and acquire $2 \pi m$ radians of phase per azimuthal revolution, are the modes of a confocal cavity of length $l$ when $z_{R}=l / 2$ and $z=0$ lies at the centre of the cavity; we label these modes $\mathscr{E}_{p m}^{(0)}$ and the associated waist parameter $w(0)=w_{0}$.

A coaxial Gaussian beam $\mathscr{E}_{00}^{(1)}(r, z)$, whose waist $w_{1} \neq w_{0}$ also lies at the centre of the cavity, may because of its cylindrical symmetry be written as a superposition of the cavity modes for which $m=0$,
$\mathscr{E}_{00}^{(1)}(r, z)=\sum_{p} a_{p} \mathscr{E}_{p 0}^{(0)}(r, z)$

The coefficients $a_{p}$ are given simply by

$a_{p}=\frac{2}{\alpha+\alpha^{-1}}\left(\frac{\alpha-\alpha^{-1}}{\alpha+\alpha^{-1}}\right)^{p}$,

where $\alpha=w_{0} / w_{1}$. Clearly, a beam of waist $w_{2}=\alpha w_{0}$ will have the same coefficients $a_{p}$ apart from a sign-changing phase term $(-1)^{p}$. Such a term is indeed introduced with each half roundtrip of the cavity, as a result of the Gouy phase shift $2 \exp (\mathrm{i}(2 p+|m|+1) \pi / 4)$; the cavity mirror thus re-focusses the Gaussian beam to a waist $w_{2}$ at the cavity centre, as we would expect from a more conventional treatment of Gaussian beam propagation, and the difference between forward and backward travelling beams destroys the exact cancellation that prevented confinement. The second cavity mirror causes the original beam to be reproduced after a complete round trip.

The two beams, equal in power, are of equal intensity at a radius $r_{0}(z)$ given by

$r_{0}(z)^{2}=\ln \frac{w_{1}(z)}{w_{2}(z)} /\left(\frac{1}{w_{2}(z)^{2}}-\frac{1}{w_{1}(z)^{2}}\right)$.

The trapping regions thus form a series of coaxial rings, spaced by $\lambda / 2$. The lowest part of the confining potential is greatest at $r(z)=\sqrt{3} r_{0}(z)$, and the trap depth near the cavity centre is maximum when $\alpha=0.492$, corresponding to 0.126 times what the central intensity would be if the beam power were focussed to a waist radius of $w_{0}$; in such circumstances, $99 \%$ of the circulating power is contained within the first 5 transverse mode components. The trap minima lie on a surface which falls within the $1 / e^{2}$ surface of the Gaussian mode for the same cavity ( $r_{0}$ varies between 0.67 $w_{0}(z)$ and $\left.0.71 w_{0}(z)\right)$. The intensity distribution around the centre of an ideal confocal cavity is shown in Fig. 2.

As an illustration, and to demonstrate the insensitivity to misalignment, we consider the trapping of atomic ${ }^{85} \mathrm{Rb}$, using a $100 \mathrm{~mm}$ long cavity with only $99 \%$ reflecting mirrors to enhance the beam from a $780 \mathrm{~nm}$ diode laser. With an incident power of $100 \mathrm{~mW}$, giving a circulating power of 


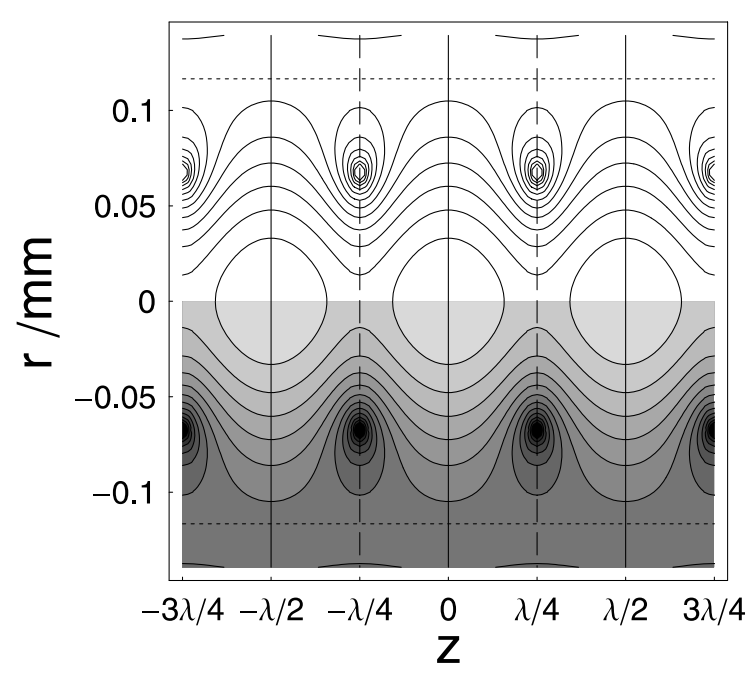

Fig. 2. The intensity distribution around the centre of a perfectly confocal resonator. Here, we have $l=100 \mathrm{~mm}, \lambda=780$ $\mathrm{nm}$, and the optimum value of $\alpha=0.492$. The dashed and solid lines indicate the nodal and antinodal planes; the dotted line shows the radius at which the lowest part of the trap wall is maximum. Contours are logarithmic, with four per decade, referred to the peak intensity on axis. The bright and dark regions are indicated schematically in the lower half of the figure.

$10 \mathrm{~W}$ and an optimal detuning [23] of $0.006 \mathrm{~nm}$, we obtain a trap depth of $0.1 \mathrm{~K}$, which is already deeper than many suggested arrangements despite the very modest finesse assumed. Resonators have been constructed with finesses in excess of a million, so in principle trap depths approaching $10 \mathrm{~K}$ would be possible with the same geometry. Furthermore, the waist radius in this example of 0.1 $\mathrm{mm}$ is considerably larger than in many dipole traps; reducing the cavity length would increase the beam intensity and hence the trap depth.

Analysis in terms of Laguerre-Gaussian cavity modes allows the effect of finite mirror reflectivity and misadjustment to be determined. For a cavity of length $l^{\prime}=l+\Delta l$, at a wavelength for which the lowest order mode $\mathscr{E}_{00}^{(0)}$ is resonant, the Gouy shift per half round trip relative to that for a confocal cavity will be given by

$\phi_{p m}=2(2 p+|m|)\left\{\tan ^{-1}\left(\frac{l^{\prime}}{2 z_{R}}\right)-\frac{\pi}{4}\right\}$.

For mirror amplitude reflection and transmission coefficients $r_{1,2}$ and $t_{1,2}$, the forward and backward travelling fields of the circulating modes at the cavity centre may be written in terms of the components of the incident beam as [32]

$\mathscr{E}_{p}^{+}(0,0)=\frac{t_{1} \exp \mathrm{i} \phi / 2}{1-r_{1} r_{2} \exp 2 \mathrm{i} \phi} a_{p} \mathscr{E}_{p}^{(0)}(0,0)$,

$\mathscr{E}_{p}^{-}(0,0)=\frac{t_{1} r_{2} \exp 3 \mathrm{i} \phi / 2}{1-r_{1} r_{2} \exp 2 \mathrm{i} \phi} a_{p} \mathscr{E}_{p}^{(0)}(0,0)$.

In Fig. 3, we show the intensity distribution around the centre of a cavity whose $99 \%$ reflecting mirrors are $0.1 \%$ from their confocal separation. The nodal surfaces are curved, reflecting the increase in Gouy phase with mode number, but the rings of minimum intensity are qualitatively little changed: the trap centre and wall maxima occur at radii around $10 \%$ greater, and the central and trapping intensities are reduced by about a third. The adjustment precision required will of course increase with the resonator finesse.

Provided that the laser wavelength is locked to the lowest order resonant mode such a cavity could be readily adjusted by observing the transmitted beam while the mirror spacing is varied, as indicated in Fig. 1. An image of the cavity centre should show a clear change in beam radius as modes are added to the superposition.

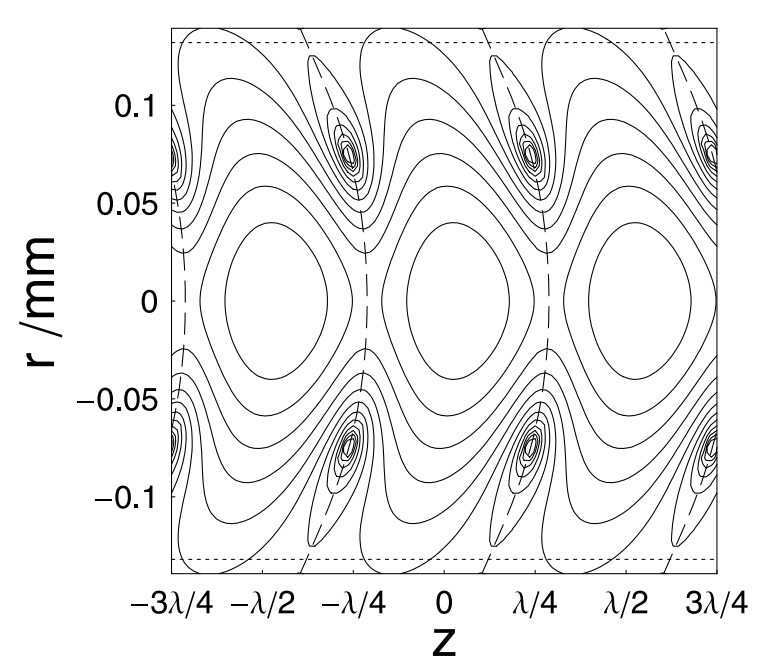

Fig. 3. Intensity distribution when the cavity mirrors are $0.1 \mathrm{~mm}$ from their confocal separation $(\Delta l / l=0.001)$, for $r^{2}=0.99, t^{2}=0.01$. 


\section{Conclusion}

We have shown that optical fields suitable for dipole force trapping of dark-field seeking atoms and molecules may be enhanced by a confocal cavity that is simultaneously resonant for all of the component modes. The Gouy phase introduces a difference - readily apparent using geometrical optics - between forward and backward travelling beams that eliminates the nodal planes of complete cancellation, along which the dark-field seeking species would otherwise escape.

We have considered a perhaps counter-intuitive example in which a single Gaussian beam is used to generate a series of dark coaxial rings spaced half a wavelength apart. These toroidal microtraps could be interesting for the study of vortices in Bose-Einstein condensates and the weak interactions between trapped species for quantum computing, while the trap overall would be suitable for confining spectrally complex molecular species, whose detection could also be enhanced by the cavity. Cooling of the trapped species could be accomplished evaporatively, by gentle reduction of the laser intensity, or through the interaction of the sample with the cavity light field.

Cavity-enhanced traps thus offer not only deeper potentials and longer storage times for a given laser power, but also a mechanism for the production of unusually shaped trapping potentials. The example described in this paper forms the basis of a generation of more advanced cavity-enhanced traps, to which we shall address future analysis.

\section{Acknowledgements}

The authors are grateful for support from the European Commission and the UK Engineering and Physical Sciences Research Council in the course of this work.

\section{References}

[1] C.S. Adams, E. Riis, Prog. Quantum Electron. 21 (1997) 1.
[2] R. Grimm, M. Weidemüller, Y.B. Ovchinnikov, Adv. At. Mol. Opt. Phys. 42 (2000) 95.

[3] S.L. Rolston, C. Gerz, K. Helmerson, P.S. Jessen, P.D. Lett, W.D. Phillips, R.J. Spreeuw, C.I. Westbrook, Proc. SPIE 1726 (1992) 205.

[4] J.D. Miller, R.A. Cline, D.J. Heinzen, Phys. Rev. A 47 (1993) R4567.

[5] S. Chu, J.E. Bjorkholm, A. Ashkin, A. Cable, Phys. Rev. Lett. 57 (1986) 314.

[6] M.D. Barrett, J.A. Sauer, M.S. Chapman, Phys. Rev. Lett. 87 (2001) 010404.

[7] S. Kuhr, W. Alt, D. Schrader, M. Müller, V. Gomer, D. Meschede, Science 293 (2001) 278.

[8] T. Takekoshi, B.M. Patterson, R.J. Knize, Phys. Rev. Lett. 81 (1998) 5105.

[9] R. Ozeri, L. Khaykovich, N. Davidson, Phys. Rev. A 59 (1999) R1759.

[10] N. Davidson, H.J. Lee, C.S. Adams, M. Kasevich, S. Chu, Phys. Rev. Lett. 74 (1995) 1311.

[11] P. Rudy, R. Ejnisman, A. Rahman, S. Lee, N.P. Bigelow, Opt. Express 8 (2001) 159.

[12] P. Zemánek, C.J. Foot, Opt. Commun. 146 (1998) 119.

[13] S.A. Webster, G. Hechenblaikner, S.A. Hopkins, J. Arlt, C.J. Foot, J. Phys. B 33 (2000) 4149.

[14] T. Kuga, Y. Torii, N. Shiokawa, T. Hirano, Y. Shimuzu, H. Sasada, Phys. Rev. Lett. 78 (1997) 4713.

[15] J. Arlt, K. Dholakia, L. Allen, M.J. Padgett, J. Mod. Opt. 45 (1998) 1231.

[16] J. Arlt, M.J. Padgett, Opt. Lett. 25 (2000) 191.

[17] J. Ye, D.W. Vernooy, H.J. Kimble, Phys. Rev. Lett. 83 (1999) 4987.

[18] S. Jochim, Th. Elsässer, A. Mosk, M. Weidemüller, R. Grimm, in: International Conference on Atomic Physics, Firenze, Italy, poster G.11, 2000;

A. Mosk, S. Jochim, H. Moritz, Th. Elsässer, M. Weidemüller, R. Grimm, Opt. Lett. (2001), to be published.

[19] P.W.H. Pinkse, T. Fischer, P. Maunz, T. Puppe, G. Rempe, J. Mod. Opt. 47 (2000) 2769.

[20] J. Yin, Y. Zhu, W. Jhe, Z. Wang, Phys. Rev. A 58 (1998) 509.

[21] X. Xu, V.G. Minogin, K. Lee, Y. Wang, W. Jhe, Phys. Rev. A 60 (1999) 4796.

[22] Y. Song, D. Milam, W.T. Hill, Opt. Lett. 24 (1999) 1805.

[23] J. Arlt, T. Hitomi, K. Dholakia, Appl. Phys. B 71 (2000) 549.

[24] P. Münstermann, T. Fischer, P. Maunz, P.W.H. Pinkse, G. Rempe, Phys. Rev. Lett. 84 (2000) 4068.

[25] N. Schlosser, G. Reymond, I. Protsenko, P. Grangier, Nature 411 (2001) 1024.

[26] T. Zaugg, M. Wilkens, P. Meystre, G. Lenz, Opt. Commun. 97 (1993) 189.

[27] M. Gangl, H. Ritsch, Phys. Rev. A 61 (1999) 011402.

[28] V. Vuletic, S. Chu, Phys. Rev. Lett. 84 (2000) 3787. 
[29] E.M. Wright, J. Arlt, K. Dholakia, Phys. Rev. A 63 (2000) 013608.

[30] J. Ye, L.-S. Ma, J.L. Hall, J. Opt. Soc. Am. B 15 (1998) 6.
[31] P. Domokos, M. Gangl, H. Ritsch, Opt. Commun. 185 (2000) 115.

[32] A.E. Siegman, Lasers, University Science Books, California, 1986 (section 11.3). 